\title{
An Explanation of the Newman-Janis Algorithm
}

\author{
S. P. Drake and Peter Szekeres \\ Department of Physics and Mathematical Physics, \\ University of Adelaide, Adelaide, \\ S. A. 5005, \\ Australia
}

February 7, 2008

\begin{abstract}
After the original discovery of the Kerr metric, Newman and Janis showed that this solution could be "derived" by making an elementary complex transformation to the Schwarzschild solution. The same method was then used to obtain a new stationary axisymmetric solution to Einstein's field equations now known as the Kerr-newman metric, representing a rotating massive charged black hole. However no clear reason has ever been given as to why the Newman-Janis algorithm works, many physicist considering it to be an ad hoc procedure or "fluke" and not worthy of further investigation. Contrary to this belief this paper shows why the Newman-Janis algorithm is successful in obtaining the Kerr-Newman metric by removing some of the ambiguities present in the original derivation. Finally we show that the only perfect fluid generated by the Newman-Janis algorithm is the (vacuum) Kerr metric and that the only Petrov typed D solution to the Einstein-Maxwell equations is the Kerr-Newman metric.
\end{abstract}

PACS numbers: 02.30.Dk, 04.20.Cv, 04.20.Jb, 95.30.Sf 


\section{Introduction}

The generation of axisymmetric solutions to Einstein's field equations is a problem which has plagued physicists for many years. The unique class of charged rotating black hole are described by Kerr-Newman spacetimes, which can be generated by a technique known as the Newman-Janis algorithm (NJA) [1] [2]. While this algorithm is particularly successful for "deriving" the Kerr metric and its electromagnetic generalization, the KerrNewman metric, it has often been criticized [3] on the grounds that (a) the procedure is not a general method of generating vacuum from vacuum metrics, and (b) there is a certain arbitrariness in the choice of complexification of terms in the original seed metric (Schwarzschild or Reissner-Nordström).

Since the Kerr metric describes the spacetime of a rotating black hole, it is naturally of interest whether or not it describes the exterior of an extended axisymmetric rotating massive body. While Birkhoff's theorem [4 tells us the spacetime exterior to a spherical symmetric massive body is uniquely described by the Schwarzschild metric there is unfortunately no reason to expect that the spacetime exterior to an arbitrary stationary axisymmetric perfect fluid body should be the Kerr spacetime. In fact it remains an open question whether the Kerr solution can represent the exterior of any perfect fluid source at all.

The first genuine example of a Kerr interior solution appears to have been provided by Neugebauer and Meinel [5], but it is rather difficult to access analytically, and as it represents a disc of matter the concept of perfect fluid can only apply in a degenerate sense. It would therefore be particularly attractive to generate interior Kerr solutions by some simple procedure, and the obvious candidate appears to be a generalization of the NJA, since that procedure is precisely capable of generating the Kerr metric from the Schwarzschild metric. The possibility of such a generalization is given some encouragement by Drake and Turolla's investigation [6] of stationary axisymmetric metrics generated by the NJA which match smoothly to the Kerr metric. The original intention of this work was to generate new metrics which could be considered as perfect fluid sources of the Kerr metric by application of the NJA to static spherical symmetric seed metrics. A class of metrics were found which match smoothly to the Kerr metric, and one particular test solution from this family was shown after detailed examination to be a "physically sensible" perfect fluid in the non-rotating limit. 
The main purpose of this paper is (a) to specify precisely what the Newman-Janis algorithm is, (b) to understand under what circumstances and with what choice of complexifications it will be successful in generating one solution of Einstein's equations from another, and (c) to explore the possibility of generalizing the algorithm to arbitrary seed metrics with the view to generating perfect fluid interior solutions of einstein's equations.

One key result from this paper is the demonstration that while the NJA is successful in generating metrics which match smoothly to the Kerr metric and are "physically sensible" perfect fluids in the zero rotation limit these metrics are not rotating perfect fluids except in the vacuum case $(P=\rho=0)$.

In Section 2 we describe the Newman-Janis Algorithm as a five-step procedure. This may appear to be somewhat overspecific, but these steps constitute the most general algorithm of this kind which has actually been found to work. In Section 3 the algorithm is applied to a general spherically symmetric spacetime. Section 4 presents the main results, that non-vacuum perfect fluids can never be generated by the NJA, while the Kerr-Newman solution is the most general algebraically special spacetime which can be so generated. It follows as a corollary that the Drake-Turolla metrics can never provide a perfect fluid interior to the Kerr metric. In Section 5 the conclusion is drawn that the particular choice of complexification used in the standard NJA to generate the Kerr-Newman solution are not arbitrary, but could in fact be chosen in no other way in order for the NJA to be successful at all. This provides, in a sense, an "explanation" of the algorithm.

\section{The Newman-Janis algorithm}

In this section we will describe the Newman-Janis algorithm in a form generalized from the original version used to generate the Kerr-Newman metric (rotating charged black hole) from the Reissner-Nordström solution. We treat the NJA as a five-step procedure for generating new solutions of Einstein's equations from known static spherically symmetric ones. Whether a similar process can apply to original seed metrics which are not spherically symmetric is not known.

The five steps of the Newman-Janis algorithm are as follows:

1. Write a static spherically symmetric seed line element in advanced null 
coordinates $\{u, r, \theta, \phi\}$

$$
d s^{2}=e^{2 \Phi(r)} d u^{2}+e^{\Phi(r)+\lambda(r)} d u d r-r^{2}\left(d \theta^{2}+\sin ^{2} \theta d \phi^{2}\right)
$$

In the Newman-Janis algorithm the seed was the Reissner-Nordström metric which in advanced Eddington-Finkelstein coordinates is

$$
d s^{2}=\left(1-\frac{2 m}{r}-\frac{Q^{2}}{r^{2}}\right) d u^{2}+2 d u d r-r^{2}\left(d \theta^{2}+\sin ^{2} \theta d \phi^{2}\right) .
$$

2. Express the contravariant form of the metric in terms of a null tetrad,

$$
g^{\mu \nu}=l^{\mu} n^{\nu}+l^{\nu} n^{\mu}-m^{\mu} \bar{m}^{\nu}-m^{\nu} \bar{m}^{\mu},
$$

where

$$
l_{\mu} l^{\mu}=m_{\mu} m^{\mu}=n_{\mu} n^{\mu}=0, \quad l_{\mu} n^{\mu}=-m_{\mu} \bar{m}^{\mu}=1, \quad l_{\mu} m^{\mu}=n_{\mu} m^{\mu}=0 .
$$

For the spacetime (11) the null tetrad vectors are

$$
\begin{aligned}
l^{\mu} & =\delta_{1}^{\mu} \\
n^{\mu} & =e^{-\lambda(r)-\Phi(r)} \delta_{0}^{\mu}-\frac{1}{2} e^{-2 \lambda(r)} \delta_{1}^{\mu} \\
m^{\mu} & =\frac{1}{\sqrt{2} r}\left(\delta_{2}^{\mu}+\frac{i}{\sin \theta} \delta_{3}^{\mu}\right) .
\end{aligned}
$$

It is also convenient to use the tetrad notation introduced by Newman and Penrose [7]

$$
Z_{a}^{\mu}=\left(l^{\mu}, n^{\mu}, m^{\mu}, \bar{m}^{\mu}\right), \quad a=1,2,3,4 .
$$

The null tetrad vectors for the Reissner-Nordström metric are

$$
\begin{aligned}
l^{\mu} & =\delta_{1}^{\mu} \\
n^{\mu} & =\delta_{0}^{\mu}-\frac{1}{2}\left(1-\frac{2 m}{r}-\frac{Q^{2}}{r^{2}}\right) \delta_{1}^{\mu} \\
m^{\mu} & =\frac{1}{\sqrt{2} r}\left(\delta_{2}^{\mu}+\frac{i}{\sin \theta} \delta_{3}^{\mu}\right) .
\end{aligned}
$$


3. Extend the coordinates $x^{\rho}$ to a new set of complex coordinates $\tilde{x}^{\rho}$

$$
x^{\rho} \rightarrow \tilde{x}^{\rho}=x^{\rho}+i y^{\rho}\left(x^{\sigma}\right),
$$

where $y^{\rho}\left(x^{\sigma}\right)$ are analytic functions of the real coordinates $x^{\sigma}$, and simultaneously let the null tetrad vectors $Z_{a}^{\mu}$ undergo a transformation

$$
Z_{a}^{\mu}\left(x^{\rho}\right) \rightarrow \tilde{Z}_{a}^{\mu}\left(\tilde{x}^{\rho}, \overline{\tilde{x}}^{\rho}\right) .
$$

Finally we require that the transformation recovers the old tetrad and metric when $\tilde{x}^{\rho}=\overline{\tilde{x}}^{\rho}$. In summary the effect of this "tilde transformation" is to create a new metric whose components are (real) functions of complex variables,

$$
g_{\mu \nu} \rightarrow \tilde{g}_{\mu \nu}: \tilde{\mathbf{x}} \times \tilde{\mathbf{x}} \mapsto \mathbb{R},
$$

while

$$
\left.\tilde{Z}_{a}^{\mu}\left(\tilde{x}^{\rho}, \overline{\tilde{x}}^{\rho}\right)\right|_{\tilde{\mathbf{x}}=\overline{\tilde{x}}}=Z_{a}^{\mu}\left(x^{\rho}\right)
$$

The tilde transformation is clearly not unique as there are many different choices of the null tetrad vector coefficients which satisfy the conditions (5) and (6).

In the original NJA, the tilde transformation on the Reissner-Nordström null tetrad vectors is

$$
\begin{aligned}
l^{\mu} & \rightarrow \tilde{l}^{\mu}=\delta_{1}^{\mu} \\
n^{\mu} & \rightarrow \tilde{n}^{\mu}=\delta_{0}^{\mu}-\frac{1}{2}\left(1-m\left(\frac{1}{\tilde{r}}+\frac{1}{\tilde{\tilde{r}}}\right)-\frac{Q^{2}}{\tilde{r} \tilde{\tilde{r}}}\right) \delta_{1}^{\mu} \\
m^{\mu} & \rightarrow \tilde{m}^{\mu}=\frac{1}{\sqrt{2} \tilde{r}}\left(\delta_{2}^{\mu}+\frac{i}{\sin \tilde{\theta}} \delta_{3}^{\mu}\right) .
\end{aligned}
$$

A quick check shows that the above null tetrad vectors are those corresponding to the Reissner-Nordström metric when $\tilde{x}^{\rho}=\overline{\tilde{x}}^{\rho}$. However it is precisely here that a certain arbitrariness crept into the process, since the method of complexifying the term $2 \mathrm{~m} / \mathrm{r}$ is quite different to the complexification of the $Q^{2} / r^{2}$ term. It is our aim to provide some rationale for this part of the NJ procedure. 
4. A new metric is obtained by making a complex coordinate transformation

$$
\tilde{x}^{\rho}=x^{\rho}+i \gamma^{\rho}\left(x^{\sigma}\right)
$$

to the null tetrad vectors $\tilde{Z}_{a}^{\mu}$. The null tetrad vectors transform in the usual way

$$
Z_{a}^{\mu}=\tilde{Z}_{a}^{\nu} \frac{\partial x^{\mu}}{\partial \tilde{x}^{\nu}}
$$

The particular choice of complex transformations chosen by Newman and Janis to generate the Kerr-Newman metric were

$$
\tilde{x}^{\rho}=x^{\rho}+i a \cos x^{2}\left(\delta_{0}^{\rho}-\delta_{1}^{\rho}\right) .
$$

From the transformed null tetrad vectors a new metric is recovered using (3). For the null tetrad vectors given by Equations (7) to (9) and the transformation given by (11) the new metric with coordinates $x^{\rho}=\{u, r, \theta, \phi\}$ in covariant form is

$$
g_{\mu \nu}=\left(\begin{array}{cccc}
1-\frac{2 m r-Q^{2}}{\Sigma} & 1 & 0 & a \sin ^{2} \theta \frac{2 m r-Q^{2}}{\Sigma} \\
\cdot & 0 & 0 & -a \sin ^{2} \theta \\
\cdot & \cdot & -\Sigma & 0 \\
\cdot & \cdot & \cdot & -\sin ^{2} \theta\left(r^{2}+a^{2}-a^{2} \sin ^{2} \theta \frac{2 m r-Q^{2}}{\Sigma}\right)
\end{array}\right) .
$$

As the metric is symmetric the "." is used to indicate $g_{\mu \nu}=g_{\nu \mu}$.

$$
\Sigma \equiv r^{2}+a^{2} \cos \theta^{2}
$$

5. Finally it is assumed that a simple coordinate transformation of the form $u=t+F(r), \phi=\psi+G(r)$ will transform the metric to Boyer-Lindquist coordinates. In this paper a set of coordinates in which the metric has only one off-diagonal term $g_{t \phi}$ will be termed "BoyerLindquist".

To obtain the usual representation of the Kerr metric in Boyer-Lindquist coordinates, it is necessary to make a transformation on the null coordinate $u$ and the angle coordinate $\phi$

$$
u=t-\int \frac{a}{r^{2}+a^{2}+Q^{2}-2 m r} d r
$$




$$
\phi=\psi-\int \frac{r^{2}+a^{2}}{r^{2}+a^{2}+Q^{2}-2 m r} d r
$$

\section{Extending the Newman-Janis algorithm}

In the various stages of the NJA described above the only ambiguous point was the tilde transformation in step 3. Applying this step to a general static spherically symmetric seed metric (I) , the tilde operation produces the null tetrad vectors

$$
\begin{aligned}
\tilde{l}^{\mu} & =\delta_{1}^{\mu} \\
\tilde{n}^{\mu} & =e^{-\lambda(\tilde{r}, \overline{\tilde{r}})-\phi(\tilde{r}, \overline{\tilde{r}})} \delta_{0}^{\mu}-\frac{1}{2} e^{-2 \lambda(\tilde{r}, \bar{r})} \delta_{1}^{\mu} \\
\tilde{m}^{\mu} & =\frac{1}{\sqrt{2} \tilde{\tilde{r}}}\left(\delta_{2}^{\mu}+\frac{i}{\sin \tilde{\theta}} \delta_{3}^{\mu}\right) .
\end{aligned}
$$

A new set of null tetrad vectors, and hence a new metric, results after the transformation (11). These new null tetrad vectors are

$$
\begin{aligned}
l^{\mu} & =\delta_{1}^{\mu} \\
n^{\mu} & =e^{-\lambda(r, \theta)-\phi(r, \theta)} \delta_{0}^{\mu}-\frac{1}{2} e^{-2 \lambda(r, \theta)} \delta_{1}^{\mu} \\
m^{\mu} & =\frac{1}{\sqrt{2}(r+i a \cos \theta)}\left(i a \sin \theta\left(\delta_{0}^{\mu}-\delta_{1}^{\mu}\right)+\delta_{2}^{\mu}+\frac{i}{\sin \theta} \delta_{3}^{\mu}\right)
\end{aligned}
$$

The coordinates $x^{\rho}=\{u, r, \theta, \phi\}$ are all real. By equation (3) the metric obtained from the null tetrad vectors (15), (16) and (17) is, in covariant form,

$$
g_{\mu \nu}=\left(\begin{array}{cccc}
e^{2 \Phi(r, \theta)} & e^{\lambda(r, \theta)+\Phi(r, \theta)} & 0 & a \sin ^{2} \theta e^{\Phi(r, \theta)}\left(e^{\lambda(r, \theta)}-e^{\Phi(r, \theta)}\right) \\
\cdot & 0 & 0 & -a e^{\Phi(r, \theta)+\lambda(r, \theta)} \sin ^{2} \theta \\
\cdot & \cdot & -\Sigma & 0 \\
\cdot & \cdot & \cdot & -\sin ^{2} \theta\left(\Sigma+a^{2} \sin ^{2} \theta e^{\Phi(r, \theta)}\left(2 e^{\lambda(r, \theta)}-e^{\Phi(r, \theta)}\right)\right)
\end{array}\right)
$$

This completes steps 3 and 4, the generalization of the NJA without guessing the tilde transformation (田). At this stage the metric contains two unknown functions $\exp (\Phi)$ and $\exp (\lambda)$ of two variables $r, \theta$. The only constraints on these functions are given by (5) and (6). 
Step 5 is the transformation of the new metric into Boyer-Lindquist coordinates by means of a transformation of the form $u=t+\int g(r) d r$ and $\phi=\psi+\int h(r) d r$ where the functions $g(r)$ and $f(r)$ will necessarily satisfy the equations

$$
\begin{aligned}
& g(r)=-\frac{e^{\lambda(r, \theta)}\left(\Sigma+a^{2} \sin ^{2} \theta e^{\lambda(r, \theta)+\Phi(r, \theta)}\right)}{e^{\Phi(r, \theta)}\left(\Sigma+a^{2} \sin ^{2} \theta e^{2 \lambda(r, \theta)}\right)} \\
& h(r)=-\frac{a e^{2 \lambda(r, \theta)}}{\sum+a^{2} \sin ^{2} \theta e^{2 \lambda(r, \theta)}} .
\end{aligned}
$$

After some algebraic manipulations one finds that in these coordinates $\{t, r, \theta, \psi\}$ the metric is

$$
g_{\mu \nu}=\left(\begin{array}{cccc}
e^{2 \phi(r, \theta)} & 0 & 0 & a \sin ^{2} \theta e^{\phi(r, \theta)}\left(e^{\lambda(r, \theta)}-e^{\phi(r, \theta)}\right) \\
. & -\Sigma /\left(\Sigma e^{-2 \lambda(r, \theta)}+a^{2} \sin ^{2} \theta\right) & 0 & 0 \\
. & \cdot & -\Sigma & 0 \\
. & . & . & -\sin ^{2} \theta\left(\Sigma+a^{2} \sin ^{2} \theta e^{\phi(r, \theta)}\left(2 e^{\lambda(r, \theta)}-e^{\phi(r, \theta)}\right)\right)
\end{array}\right) .
$$

By rearrangement of equation (20) we find

$$
e^{2 \lambda(r, \theta)}=\frac{-h(r) \Sigma}{a^{2} h(r) \sin ^{2} \theta+a}=\frac{\Sigma}{j(r)+a^{2} \cos ^{2} \theta} .
$$

where $j(r) \equiv-a / h(r)-a^{2}$.

In a similar manner equation (19) may be used to express $\exp \Phi(r, \theta)$ in terms of the single variable functions $g(r)$ and $j(r)$,

$$
e^{\Phi(r, \theta)}=\frac{\sqrt{\sum\left(j(r)+a^{2} \cos ^{2} \theta\right)}}{k(r)+a^{2} \cos ^{2} \theta}
$$

where $k(r) \equiv-g(r)\left(j(r)+a^{2}\right)-a^{2}$. The Boyer-Lindquist form of (18) is then

$$
g_{\mu \nu}=\left(\begin{array}{cccc}
\frac{\Sigma\left(j(r)+a^{2} \chi^{2}\right)}{\left(k(r)+a^{2} \chi^{2}\right)^{2}} & 0 & 0 & -\frac{a\left(1-\chi^{2}\right)(j(r)-k(r)) \Sigma}{\left(k(r)+a^{2} \chi^{2}\right)^{2}} \\
\cdot & -\frac{\Sigma}{j(r)+a^{2}} & 0 & 0 \\
\cdot & \cdot & -\frac{\Sigma}{1-\chi^{2}} & 0 \\
\cdot & \cdot & \cdot & -\left(1-\chi^{2}\right) \Sigma \frac{\left(k(r)+a^{2}\right)^{2}-a^{2}\left(1-\chi^{2}\right)\left(j(r)+a^{2}\right)}{\left(k(r)+a^{2} \chi^{2}\right)^{2}}
\end{array}\right) .
$$


Where $\chi \equiv \cos \theta$ so that

$$
\Sigma \equiv r^{2}+a^{2} \chi^{2}
$$

In order to calculate properties of the metric tensor (22) the packages Tensor and Debever where used inside Maple $V$. It is a well known phenomena that while humans often prefer to work with trigonometric functions computers do not. The cause of this problem is that there is not a unique way to simplifying trigonometric functions. The safest way to remove this problem is to avoid using trigonometric functions altogether in computer aided calculations. For this reason the substitution $\chi \equiv \cos \theta$ was made.

\section{Properties of metrics generated by the Newman- Janis algorithm}

The package Tensor in Maple $V$ allows us to calculate the Einstein tensor any metric tensor. Debever calculates the Newman-Penrose spin coefficients. Below, we provide some theorems for these spacetimes using the above mentioned packages. The algebraic expressions of the Einstein tensor and the spin coefficients tend to be rather lengthy, fortunately all those of interest to us can be expressed in the form $\sum_{m} H_{m} \chi^{2 m}$ where $H_{m}$ is a function of $r$ only. For reasons of compactification all the curvature expressions will be written in this way and the specific forms for $H_{m}$ will be shown only when required. The interested reader is encouraged to check these expressions of Maple, Mathematica or their favorite algebraic manipulation program.

Theorem 1 The only perfect fluid generated by the Newman-Janis Algorithm is the vacuum.

Proof: The Einstein tensor resulting from the metric (22) has two off diagonal terms, $G_{t \Phi}$ and $G_{r \chi}$. If the Einstein tensor is equivalent to the stress energy tensor of a perfect fluid

$$
G_{\mu \nu}=(P+\rho) U_{\mu} U_{\nu}-P g_{\mu \nu}
$$

then it is required that $G_{r \chi}=0$. The reason for this is that the four velocity $U_{\nu}$ being a time-like vector must have $U_{0} \neq 0$. Generation of the Einstein tensor reveals $G_{t A}=0$ if $A=r$ or $A=\chi$. From equations (22) and (23) it 
follows that $U_{r}=U_{\chi}=0$. Since $g_{t \phi}$ is the only non-zero component of $g_{\mu \nu}$ it follows that $G_{r \chi}$ must vanish identically. The $G_{r \chi}$ component of the Einstein tensor generated by Tensor in Maple $V$ is

$$
-3 \chi a^{2} \frac{\left(2 r-k(r)^{\prime}\right) a^{4} \chi^{4}+\left(2 k(r)-k(r)^{\prime} r\right) 2 a r \chi^{2}-k(r)^{\prime} r^{4}+2 r k(r)^{2}}{\Sigma^{2}\left(k(r)+a^{2} \chi^{2}\right)^{2}},
$$

where " " " denotes the derivative with respect to $r$. This expression vanishes if and only if

$$
k(r)=r^{2} .
$$

To resolve $j(r)$ with this definition of $k(r)$ we look at the isotropic pressure condition,

$$
\begin{aligned}
G_{r r} / g_{r r} & -G_{\chi \chi} / g_{\chi \chi}=0 \\
\frac{-1}{2} \frac{\left(j(r)^{\prime \prime}-2\right) a^{2} \chi^{2}}{r^{2}+a^{2} \chi^{2}} & -\frac{1}{2} \frac{2 r^{2}+r^{2} j(r)^{\prime \prime}-4 r j(r)^{\prime}+4 j(r)}{r^{2}+a^{2} \chi^{2}}=0 .
\end{aligned}
$$

As $\chi$ is an independant variable the isotropic pressure condition (25) is satisfied if and only if

$$
\begin{aligned}
j(r)^{\prime \prime} & -2=0 \\
\left.j(r)^{\prime \prime}-2\right) a^{2} \chi^{2}+2 r^{2}+r^{2} j(r)^{\prime \prime} & -4 r j(r)^{\prime}+4 j(r)=0 .
\end{aligned}
$$

The unique solution to this pair of equations is

$$
j(r)=r^{2}+d_{1} r
$$

where $d_{1}$ is a constant of integration.

Substituting equations (26) and (24) into the metric (22) generated by the NJA, and setting the constant of integration $d_{1}$ to equal twice the mass we get the Kerr metric in Boyer-Lindquist coordinates.

Theorem 2 The only algebraically special spacetimes generated by the NewmanJanis algorithm are Petrov type D.

Proof: It was shown in section of that since the metric (18) can be transformed to Boyer-Lindquist coordinates the functions $\exp \Phi(r, \theta)$ and 
$\exp \lambda(r, \theta)$ can be expressed in the form

$$
\begin{aligned}
e^{\lambda(r, \chi)} & =\frac{\sqrt{\Sigma}}{\sqrt{j(r)+a^{2} \chi^{2}}} \\
e^{\Phi(r, \chi)} & =\frac{\sqrt{\Sigma\left(j(r)+a^{2} \chi^{2}\right)}}{k(r)+a^{2} \chi^{2}} \\
\Sigma & \equiv r^{2}+a^{2} \chi^{2} \\
\chi & \equiv \cos \theta .
\end{aligned}
$$

The resulting null tetrad vectors are

$$
\begin{aligned}
l^{\mu} & =\delta_{1}^{\mu} \\
n^{\mu} & =\frac{k(r)+a^{2} \chi^{2}}{\Sigma} \delta_{1}^{\mu}-\frac{1}{2} \frac{j(r)+a^{2} \chi^{2}}{\Sigma} \delta_{0}^{\mu} \\
m^{\mu} & =\frac{1}{\sqrt{2}(r+i a \chi)}\left(i a \sqrt{1-\chi^{2}}\left(\delta_{0}^{\mu}-\delta_{1}^{\mu}\right)+\delta_{2}^{\mu}+\frac{i}{\sqrt{1-\chi^{2}}} \delta_{3}^{\mu}\right)
\end{aligned}
$$

Using the package Debever in Maple $V$ it is possible to compute the NewmanPenrose coefficients [7] from the null tetrad vectors (31), (32) and (33). It is found that $\Psi_{0}$ is identically zero. A spacetime is said to be algebraically special [8] if $\Psi_{0}=\Psi_{1}=0 . \Psi_{1}$ is a rather long expression which can however be expressed as

$$
\Psi_{1}=\frac{\sum_{m=0}^{4} i K_{m} \chi^{2 m}}{\Sigma^{2}(r-i a \chi)\left(k(r)+a^{2} \chi^{2}\right) \sqrt{1-\chi^{2}}},
$$

where $K_{m}$ are functions of $k(r)$ only. That is, the vanishing of $\Psi_{1}$ is independent of $j(r)$. As $r$ and $\chi$ are independent variables, $\Psi_{1}=0$ if and only if $K_{m}=0$ for $m=0,1,2,3,4$.

$$
K_{4}=k^{\prime \prime}(r)-2
$$

which equals zero if and only if $k(r)=r^{2}+c_{1} r+c_{0}$. Substituting this expression for $k(r)$ into $K_{3}$ it is found that $K_{3}=a^{4}\left(4 c_{0}-c 1^{2}\right)$ which equals zero for $a \neq 0$ if and only if $c_{1}^{2}-4 c_{0}=0$ so that

$$
k(r)=r^{2}+c_{1}\left(r+c_{1} / 4\right) .
$$


Furthermore with $k(r)$ given by equation (35) it is easy to show by direct substitution that $K_{m}=0$ for all allowed values of $m$.

Hence all spacetimes generated by the Newman-Janis Algorithm which are algebraically special uniquely satisfy equation (35). The proof that they also Petrov type D involves substituting the expression for $k(r)$ into the expressions for the Newman-Penrose spin coefficient $\Psi_{i}$ and showing that they satisfy the relation

$$
\Psi_{2} \Psi_{4}-2 \Psi_{3}^{2} / 3=0 .
$$

This can be checked checked with the Debever package in Maple $V$.

Theorem 3 The only Petrov type D spacetime generated by the NewmanJanis algorithm with a vanishing Ricci scalar is the Kerr-Newman spacetime.

Proof: Solutions to the Einstein-Maxwell field equations have a vanishing Ricci scalar. Once again using Tensor within Maple $V$ it is possible to calculate the Ricci scalar from the metric (22) with the definition (35). By grouping the Ricci scalar $R$ into powers of $\chi$

$$
R=\frac{\sum_{m=0}^{4} J_{m} \chi^{2 m}}{\Sigma^{3}\left(r^{2}+c_{1}\left(r+c_{1} / 4\right)\right)^{2}},
$$

$J_{m}$ are functions of $j(r)$ only. As $r$ and $\chi$ are independent variables $R=0$ if and only if $J_{m}=0$ for all allowed values of $m . J_{4}=j(r)^{\prime \prime}-2$ which equals zero if and only if $j(r)=r^{2}+d_{1} r+d_{0}$. Substituting this expression for $j(r)$ along with (24) into $R$ it is found that

$$
R=c_{1} \frac{\sum_{m=0}^{3} I_{m} \chi^{2 m}}{\Sigma^{3}\left(r^{2}+c_{1}\left(r+c_{1} / 4\right)\right)^{2}},
$$

where $I_{m}$ depends on the constants $c_{1}, d_{1}, d_{0}$ and the variable $r$. It is not difficult, though tiresome, to show that if you do not assume that $c_{1}=0$ then $c_{1}=-2 d_{1}$ and $d_{1}=d_{0}=0$. The vanishing of $R$ is assured if and only if $c_{1}=0$. In which case the functions $j(r)$ and $k(r)$ are

$$
\begin{aligned}
& j(r)=r^{2}+d_{1} r+d_{0} \\
& k(r)=r^{2}
\end{aligned}
$$

Setting the constants of integration $d_{1}$ and $d_{0}$ to be twice the mass and the square of the charge of the black hole respectively one obtains the KerrNewman metric. 


\section{Conclusion}

Previously all work on the Newman-Janis algorithm has involved some guess work. It was noticed that the Kerr-Newman metric could be obtained if a complex extension to the metric coefficients of the Reissner-Nordström seed metric was made

$d s^{2}=\left(1-m\left(\frac{1}{r}+\frac{1}{\bar{r}}\right)+\frac{Q^{2}}{r \bar{r}}\right) d t^{2}-\left(\frac{r \bar{r}}{r \bar{r}-m(r+\bar{r})+Q^{2}}\right) d r^{2}-r \bar{r} d^{2} \theta-r \bar{r} \sin ^{2} \theta d^{2} \phi$

before applying the NJA, where the bar denotes the complex conjugate of a particular variable. The only reason given for doing this was that it was successful. Our analysis does not rely on any such guess work and gives an unambiguous explanation of the success of the NJA in generating KerrNewman metric.

In this paper we have proved the following: 1) The only perfect fluid spacetime generated by applying the Newman-Janis algorithm to a static spherically symmetric seed metric which may be written in Boyer-Lindquist form is the Kerr metric. 2) The only algebraically special spacetimes generated by applying the Newman-Janis algorithm to static spherically symmetric seed metrics which may be written in Boyer-Lindquist form are Petrov-type D. 3) The only algebraically special spacetime generated by applying the Newman-Janis algorithm to a static spherically symmetric seed metric which may be written in Boyer-Lindquist form and which has vanishing Ricci scalar (e.g. is a solution of the Einstein-Maxwell equations) is the Kerr-Newman metric.

The relation of this work to the previous results of Drake and Turolla [6] is that while the NJA is successful in generating interior spacetimes which match smoothly to the Kerr metric, even if these interiors are perfect fluids in the non rotating limit this is not the case when rotation is included.

\section{Acknowledgments}

All of algebraic calculations presented in this paper were done with the aid of Maple $V$ and using the routines Tensor and Debever which are contained therein. SPD would like to thank the Australian Post-graduate Programme for support during the completion of this work. SPD would also like to thank 
D. Hartley, M. Howes, T. Rainsford, R. Turolla and D. Wiltshire for many helpful discussions.

\section{References}

[1] E. T. Newman and A. I. Janis, J. Math. Phys. 6915 (1965).

[2] E. T. Newman, E. Couch, K. Chinnapared, A. Exton, A. Prakash and R. Torrence, J. Math. Phys. 6918 (1965).

[3] E.J. Flaherty Hermitian and Kählerian geometry in relativity, Lecture notes in Physics 46 (Springer-Verlag, Berlin 976).

[4] C. W. Misner, K. S. Thorne and J. A. Wheeler, Gravitation (W.H. Freeman and Co., San Francisco, 1973).

[5] G. Neugebauer and R. Meinel, Phys. Rev. Lett. 73, 2166 (1994)

[6] S. P. Drake and R. Turolla, Class. Quantum Grav. 141883 (1997).

[7] E. T. Newman and R. Penrose, J. Math. Phys. 3566 (1962).

[8] S. W. Hawking and W. Israel, General Relativity: An Einstein Century Survey, (Cambridge University Press, Cambridge, 1979). 